\title{
The distorting effects of deciding to stop sampling information
}

\author{
Anna Coenen \& Todd Gureckis \\ New York University
}

\begin{abstract}
This paper asks how strategies of information sampling are affected by a learner's goal. Based on a theoretical analysis and two behavioral experiments, we show that learning goals have a crucial impact on the decision of when to stop sampling. This decision, in turn, affects the statistical properties (e.g. average values, or standard deviations) of the data collected under different goals. Specifically, we find that sampling with the goal of making a binary choice can introduce a correlation between the average value of a sample and its size (the number of values sampled). Across multiple rounds of sampling, this has the potential of biasing learners' inferences about the underlying process that generated the samples, specifically if learners ignore sample size when making these inferences. We find that people are indeed biased in this way and make different inferences about the same data-generating process when sampling with different learning goals. These findings highlight yet another danger of inferring general patterns from samples of evidence the learner had a hand in collecting.
\end{abstract}

Keywords: sampling, information search, optimal stopping

\section{Introduction}

Any student of statistics is aware that the way in which information is collected, or sampled, strongly influences inference. For example, a study testing the efficacy of performance enhancing diets on the basis of a convenience sample of elite athletes may have limited generalizability to the broader population. Of course, the way information is collected is not only a concern for researchers and statisticians. As we go about our lives we often have to make inductive inferences based on samples of limited information which were collected or encountered in certain ways (Fiedler \& Juslin, 2006).

Assuming we have some choice in the matter, what is the best way to collect information for subsequent inference and decision making? Fiedler (2008) describes the "ultimate sampling dilemma", which highlights how different information sampling strategies convey both advantages and disadvantages for certain types of inference and judgment.

To understand the roots of this dilemma, consider a music lover looking for new artists by exploring music online. An efficient search strategy might be to listen to particularly popular songs from each artist and decide whether or not they like their music based on that sample of songs. Such selective sampling has the crucial benefit that it allows learners to collect information that is most relevant to their current goal (find out quickly if they like an artist or not). However, it can also be the source of biases that occur when learners make more general inferences based on those samples (e.g., Denrell, 2003; Fiedler, 2000). For example, by focusing only on an artist's most popular songs, it is possible to completely miss parts of their oeuvre that happen to be less commer- cially successful but more enjoyable. This kind of selection bias is a ubiquitous phenomenon that affects inferences in many domains, such as social reasoning (Pachur, Hertwig, \& Rieskamp, 2013; Denrell, 2005), economics (Denrell, 2003), and empirical science (Francis, 2012). Unfortunately, past work has also shown that people are often unaware of how biased information selection affects their judgments and are unable to correct for its potentially harmful effects, a finding that Fiedler calls "metacognitive myopia" (e.g., in Fiedler, 2008, 2000; Unkelbach, Fiedler, \& Freytag, 2007)

An alternative information gathering strategy that can sometimes remedy selection bias is to engage in what Fiedler (2008) calls natural sampling. Unlike selective sampling, natural sampling is the process of experiencing samples directly from their generating distribution without conditioning on any other feature(s) of the world. For example, the music lover could choose to listen to an artist's songs in random order to get a picture of the entirety of their work. Natural sampling lets the learns experience the world "as it flows by." On the one hand, this approach may be less efficient than selective sampling (it might involve listening to more material before deciding if they like an artist). On the other hand, it yields a representative sample of the underlying distribution and will improve the quality of future generalizations.

Intuitively, natural sampling appears to eliminate sampling bias because it prevents a-priori censoring of encountered information. However, even with natural sampling, learners still get to decide at what point to terminate sampling. For example, the music lover will at some point stop their exploration and decide whether or not to permanently add an artist to their music library. This paper addresses how 
the choice of when to stop sampling affects subsequent inference and decision making. We will show that, similar to selective sampling, learning goals can have a strong impact on people's stopping strategies, which ultimately also affects the statistical properties of samples collected under different goals. We further investigate if this can bias people's beliefs about the underlying distribution in a similar way as has been found during selective sampling. The paper ultimately offers a warning about the dangers of not accounting for sampling strategies when performing inductive inference.

\section{Prior work on stopping decisions}

Deciding when to stop collecting information is particularly important when that information is costly in terms of time, money, or cognitive resources (Edwards, 1965; Busemeyer \& Rapoport, 1988; Juni, Gureckis, \& Maloney, 2016). A commonly posed question is whether people collect too little or too much information (i.e. if they stop too early or too late) with respect to a given cost structure (Seale \& Rapoport, 1997; Tversky \& Edwards, 1966; Juni et al., 2016; Busemeyer \& Rapoport, 1988; Hertwig \& Pleskac, 2010). To summarize, this work has provided some examples of systematic deviations from optimal stopping behavior. For example, when the costs and benefits of sampling are explicitly provided, it is often found that people collect too much information (i.e., they stop too late), compared to the utility maximizing strategy of the task (see, Tversky \& Edwards, 1966; Juni et al., 2016). In contrast, tasks without explicit costs have found that people stop collecting information seemingly early (e.g., Seale \& Rapoport, 1997). Despite such suboptimalities (driven in part by risk aversion), past work has shown that people are generally sensitive to the goals of a task and can trade off the costs and benefits of collecting more samples at least qualitatively. For instance, in a simple binary sampling task Busemeyer and Rapoport (1988) found that participants stopped earlier or later when sampling costs were increased or decreased, and additionally collected larger samples when the evidence they received was ambiguous (all behaviors broadly consistent with an optimal analysis of the stopping task). Analyses of stopping problems also play an important role in a number of cognitive models. For example, sampling-based models of memory retrieval or perceptual decision-making often assume that people trade off speed (i.e., the number of samples drawn from memory/stimulus) and accuracy and that this is measurable using reaction time (Ratcliff \& Rouder, 1998; Ratcliff, 1978; Bogacz, Hu, Holmes, \& Cohen, 2010; Bogacz, Brown, Moehlis, Holmes, \& Cohen, 2006; Nosofsky \& Palmeri, 1997).

\section{Overview of this paper}

In this paper, we offer a cautionary tale about the dangers of stopping rules on inductive inference, even under conditions of natural sampling. In particular, we will show that - similar to selective sampling - approximately optimal stopping regimes for a specific sampling goal can yield samples that have the potential to bias judgments about the overall population.

We proceed in three steps. First, we explore how different sampling goals (i.e., objectives for learning) affect people's stopping decisions. Second, we evaluate what effect such goal-induced stopping regimes have on sample characteristics, and whether samples generated by self-directed stopping leave the statistical properties of the environment intact. Third, we investigate if people can correct for potential sampling biases that result from their stopping decisions or if they are prone to a type of "metacognitive myopia" that has been demonstrated during selective sampling (Fiedler, 2008, 2000; Unkelbach et al., 2007).

\section{Optimal stopping under two sampling goals}

Throughout this article, we will compare two sampling goals (here a goal refers to an objective for inference and choice). Although there exists an infinite number of possible goals, we focus on these two because they are applicable to different types of distributions, and because they both constitute common objectives that guide everyday information search.

1. Binary Choice: A common goal of information gathering is to support binary decisions, like choosing between two options with different values (e.g., predicting which opponent will win a match), deciding whether to give a "thumbs up" or "thumbs down" rating of an online service, or determining if some value will exceed a certain threshold (e.g., "will this student get enough points to pass the test?" or "Will this party get enough votes to enter parliament?"). These decisions are particularly common when absolute differences do not matter as much as rank differences (e.g., the exact differences in finishing times do not matter for determining who wins a race).

2. Estimation: Learners also often sample their environment to estimate the value of some parameter in the world, like the mean of a distribution, an average rate of occurrence, or the probability of some event. For example, investors and consumers collect information to estimate the value of assets or products, educators seek information to assess the abilities of their students, and weather forecasters try to determine the likelihood of rainfall.

To demonstrate the impact of these goals on optimal stopping and the resulting sample composition, we will walk in detail through an example that uses outcomes from a simple Bernoulli process. In the Appendix, we also provide model predictions for the case of sampling a Gaussian process. Both 
types of distributional assumption are explored in the behavioral experiments presented below.

Optimal stopping with Bernoulli trials. Consider a simple information search task in which learners repeatedly observe binary outcomes. For example, learners could be picking either good or bad apples in order to learn something about the quality of the tree. The tree will have some probability of yielding good and bad apples, but learners have to rely on a finite sample of apples to estimate this probability.

This example can, to a first approximation, be modeled as a Bernoulli process (e.g., a coin flip) with two i.i.d. (independent and identically distributed) outcomes (heads or tails) and an stationary outcome probability $\theta$ (probability of heads). We assume that the learner incurs a small cost, $c$, for every draw from this distribution (every coin flip). We also assume that they will subsequently have to answer one of the following two questions (sampling goals).

1. Binary: Find out if $\theta$ is greater than 0.5 ("Is the coin biased towards heads or tails?").

2. Estimation: Find out the exact value of $\theta$ ("What is the bias of the coin?").

Each goal is associated with a loss function, which itself is a function of the true value of $\theta$ and the learner's response. The task is to decide when to stop sampling given the sample cost, the potential final reward, and the current sample (heads and tails seen so far). Assuming there exists a maximum number of samples that learners are allowed to draw, this task can be framed and solved as an finite-horizon $o p$ timal stopping problem. In every possible state (defined by the size of the current sample, $n$, and number of heads in that sample, $h_{n}$ ), an optimal decision maker should compare the expected value of stopping and of continuing and choose whichever is higher. Thus the expected value of a state, given a horizon of a maximum of $T$ flips is

$$
V_{n}^{(T)}\left(n, h_{n}\right)=\max \left\{V_{\text {stop }}, V_{\text {cont }}\right\}
$$

where

$$
V_{\text {stop }}=u_{\text {stop }}\left(n, h_{n}\right)-n c
$$

and

$$
V_{\text {cont }}=E\left[V_{n+1}^{(T)}\left(n+1, h_{n+1}\right)\right]
$$

and where $u_{\text {stop }}($.$) is the expected utility of the post-sampling$ task, which depends on a learner's sampling goal (see below). The value of continuing to obtain another sample is a learner's expectation over possible future states given their current knowledge. The probabilities in the expectation are based on a beta distribution parameterized by the outcomes observed so far. The value of the final state (when $n=T$ ) is just the expected value of stopping, which, along with the Markov property, means that this problem is solvable by backwards induction (Ferguson, n.d.).
To compute the utility of stopping under the binary goal, note that the probability of a coin being biased towards heads is $P(\theta>.5)=1-I_{0.5}(\alpha, \beta)$, where $I_{x}(\alpha, \beta)$ is the cumulative distribution function of the beta distribution with parameters $\alpha=h+1$ and $\beta=(n-h)+1$. Assuming that the learner chooses heads when $P(\theta>.5)>0.5$ and tails otherwise, their expected utility for stopping is

$$
u_{\text {stop }}^{\text {bin }}=\max \left\{1-I_{0.5}(\alpha, \beta), I_{0.5}(\alpha, \beta)\right\} r
$$

where $r$ is the reward for making a correct choice.

The expected utility from stopping under the estimation goal requires specifying a cost function over the distance between the participant's estimate and $\theta$. For simplicity's sake, assume that the learner's answer counts as correct whenever their response lies within a .2 interval surrounding the true value, and incorrect otherwise. The expected utility from stopping under this goal is

$$
u_{\text {stop }}^{\text {est }}=\max _{\theta}\left\{\int_{0}^{1} \operatorname{Beta}(x ; \alpha, \beta) w_{0.2}(\theta-x) d x\right\} r
$$

where $w_{0.2}$ is a boxcar function with a .2 wide interval. By convolving it with the posterior over $\theta$ it can be used to find the interval with the largest posterior density.

\section{Model Predictions}

To predict behavior using this model, we need to choose values for sampling cost, $c$, potential reward of the secondary task, $r$, and the maximum number of trials (the length of the horizon), $T$. To generate more realistic predictions, we additionally assume some stochasticity in people's choice behavior by using a probabilistic choice rule instead of the $\max ()$ operator in Equation (1). For example, using a softmax choice rule yields the following probability of stopping at each state,

$$
P_{\text {stop }}=\frac{\exp \left(V_{\text {stop }} / \tau\right)}{\exp \left(V_{\text {stop }} / \tau\right)+\exp \left(V_{\text {cont }} / \tau\right)} .
$$

It requires specifying an additional temperature parameter, $\tau$ which governs the degree of probabilistic responding (when $\tau \rightarrow 0$ it chooses the maximum value, as $\tau \rightarrow \infty$ it chooses randomly). To help derive initial intuition about these optimal strategy, we fixed the following parameter settings: $T=24, c=\$ 0.03, r=\$ 2, \tau=.02^{1}$ The model was used to derive predictions for the expected sample size, stopping probabilities, and the composition of samples after stopping.

\footnotetext{
${ }^{1}$ These values were chosen for illustrative purposes and many qualitative results hold across a broader range of values (as long as the trade-off between $c$ and $r$ leads to stopping before the horizon is reached). Note that the choice of $T$ has very little impact on predictions, as long as it is large enough to not curtail sampling sequences before they reach a point at which stopping becomes more beneficial.
} 


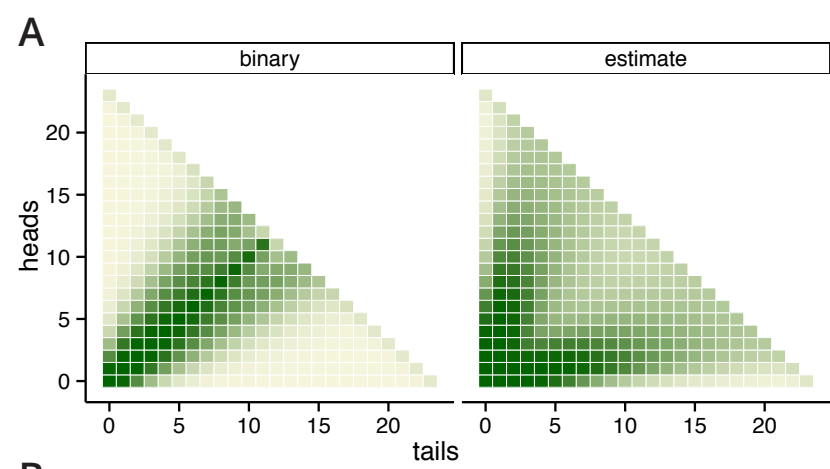

B

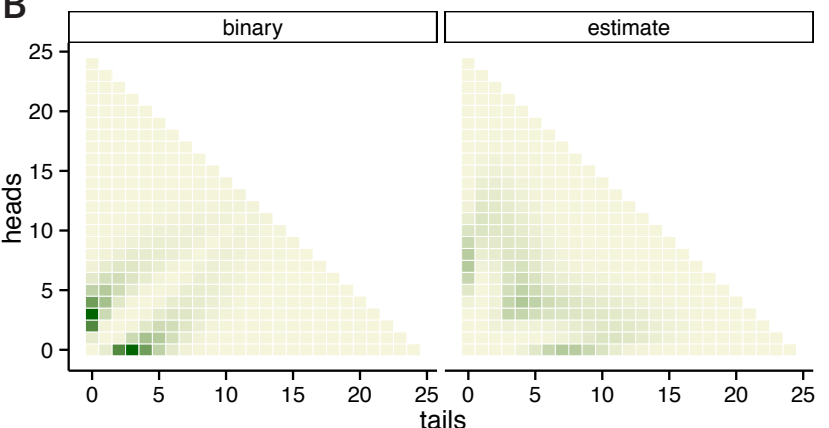

Figure 1. Model Predictions. (A) Probability of continuing (dark) or stopping (light) given observed data. (B) Probability of ending up with every possible final sample, assuming a uniform distribution over values of $\theta$.

Stopping Points. Figure 1A shows predictions for the learner's decisions to stop (light) or continue (dark) sampling given the current state (outcomes observed so far). Under the binary goal, learners should continue sampling if the current sample is balanced, in terms of the proportion of heads and tails, and be more likely to stop when it is more extreme (average closer to 0 or 1 ). In contrast, the estimation goal predicts a more similar probability of stopping in a much broader range of states. This is unsurprising because estimation requires a representative sample and overall more data than binary choice. A more subtle prediction is that the probability is not uniform for each sample size, but shows a slight pattern of earlier stopping for both very extreme and very balanced samples ${ }^{2}$. Both patterns are caused by a learner's expected success at the estimation task. When samples are extreme, expected accuracy is slightly higher and learners can stop earlier with a reasonable chance at success. When samples are very mixed (close to 0.5 average), a high chance of good performance would require too many costly samples, at which point stopping early and making a best guess has higher expected value.

From the stopping matrices in Figure 1A one can now derive expected probabilities for stopping points, that is, for the samples that learners eventually end up with after they stopped sampling. Note that this value can only be computed for specific values of $\theta$. Figure 1B shows the expectation over stopping points assuming a uniform distribution of possible values for $\theta$. As expected, binary learners often end up collecting small samples with extreme average (all heads or all tails) or larger samples with less extreme averages. Estimation learners show a wider distribution of possible final samples.

Sample Size. Figure 2A shows the expected distribution of sample sizes, assuming that learners are sampling from a processes with uniform $\theta$. Estimation learners are expected to collect larger samples than binary learners, who face an easier secondary task and therefore terminate earlier on average.

Sample Composition. The fact that stopping rules affect expected size of samples is not surprising given the loss functions of the two goals. A more intriguing question is whether sampling goals also preserve the properties of the distribution that generates each sample. To investigate this, we will consider the proportion of heads and tails within each sample, after repeatedly sampling and stopping under the two goals. We expect that sampling with a binary goal would on average lead to more unequal samples (all heads or all tails), because its stopping rule terminates early when outcomes are extreme (after two or three heads one can be pretty confident that heads is more common, for example) and continues when early outcomes are mixed (after one tails and two heads a decider might want to flip the coin at least one more time to be sure). For the binary goal, this leads to a particularly strong relationship between the size of a sample and its strengh, that is, the proportion of the more likely outcome in a given sample. Figure 2B shows this relationship for both sampling goals, again based on model predictions for uniform values of $\theta$. Indeed, sample size and strength are strongly negatively related for the binary goal, while the relationship is mostly flat for the estimation goal (except for some low odd-numbered sample size bins, which necessarily contain more high strength samples because there cannot be an even split between heads and tails in samples with odd sample sizes).

Figures $2 \mathrm{C}$ and $\mathrm{D}$ further confirm this effect of stopping rules on sample composition. They show the expected frequency distribution of sample averages under each sampling goal, when the true $\theta=0.5(\mathrm{C})$ or $\theta=0.7(\mathrm{D})$. In the estimation condition, sample means fall around the expected value of the distribution, whereas the binary condition leads to many samples with only one type of outcome (all heads or all tails). Note that these more extreme samples tend to be small (that's when learners terminate early), but they still mostly contain more than one observation, which can be seen

\footnotetext{
${ }^{2}$ Note that the same does not hold for the Gaussian sampling example which is described in the Appendix. It predicts a fixedsample size stopping rule for the estimation goal.
} 

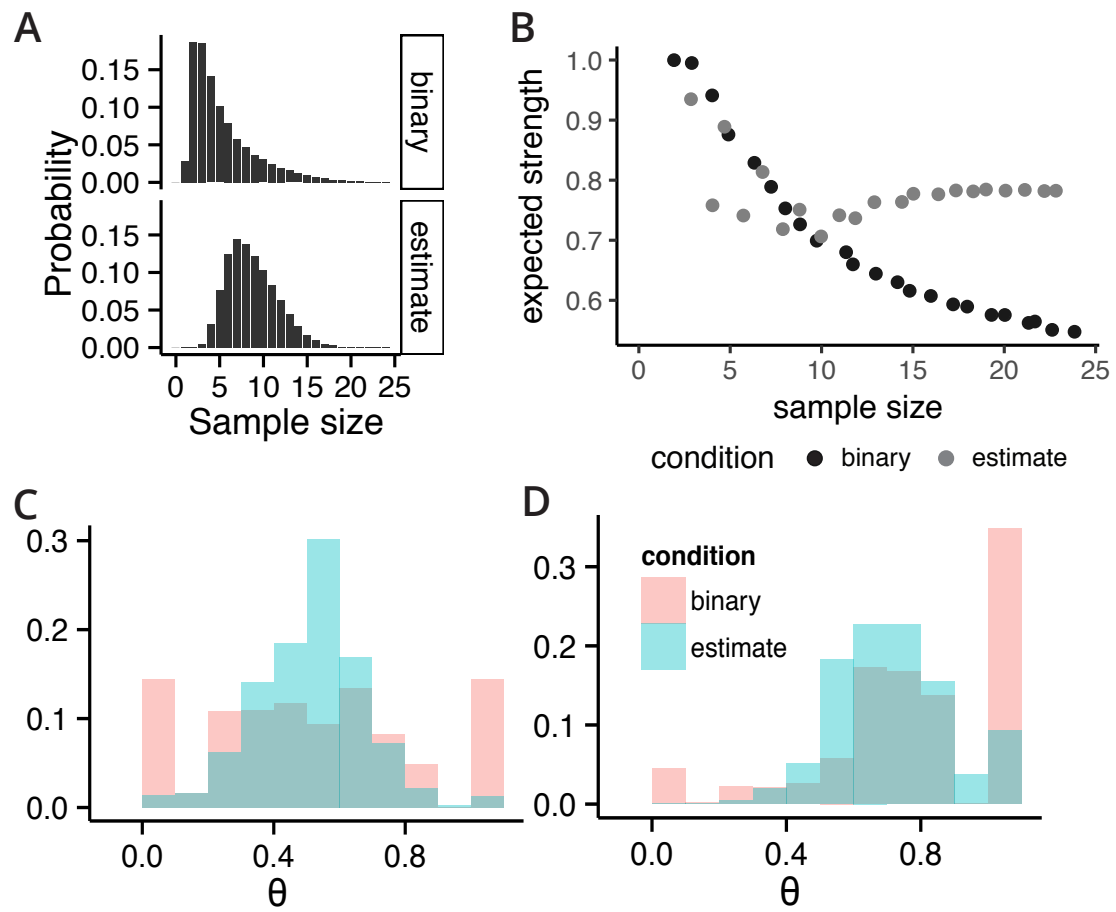

Figure 2. (A) Distribution of sample size by condition, using uniform $\theta$. (B) Predicted relationship between sample size and sample strength (proportion of more common outcome in sample). (C) and (D) Predicted distribution of sample averages, using $\theta=0.5$, and $\theta=0.7$.

from Figure 1A. Thus, the difference in sample mean distributions between the two goals is not just due to the fact that the binary condition leads to samples of size 1 which necessarily have an average of 0 or 1 .

\section{Summary of modeling results}

This model-based analysis shows how sampling goals can influence learners' expected stopping strategies in a simple Bernoulli sampling task. Crucially, it showed that, assuming samples are collected optimally with respect to the goal, the resulting distribution of samples does not "conserve the properties of the universe" (Fiedler, 2008). Instead, sampling with the binary goal leads to far more samples with extreme averages than the estimation goal. This result emerges because sampling with a binary goal introduces a negative relationship between the strength of a sample (the proportion of outcomes leaning towards an average of 0 or 1) and its size. In producing many small samples with high strength and some large samples of moderate strength, the resulting distribution of sample means looks more extreme than the true underlying distribution of outcome probabilities. In the following studies we explore the empirical consequences.

\section{Behavioral experiments}

The goal of the following experiments is to investigate how sampling goals affect stopping regimes, the resulting sample distributions, and the judgments people make based on the samples collected under different goals.

In terms of the stopping rule, we are particularly interested if the behavioral difference between sampling with a binary or an estimation goal match the predictions of the optimal analysis, including in its effect on sample composition. Specifically, we test if sampling with a binary goal yields a (negative) correlation between sample strength and sample size, as shown in the modeling analysis (see Figure 2B). Answering these questions is the main goal of Experiment 1.

Our second set of questions, which is addressed in Experiment 2, concerns judgments about the distribution that generates the samples. Since sampling with a binary goal (according to the model) produces more extreme samples, we ask if this can lead people to believe that extreme outcomes are more likely than they actually are. Based on prior work, there are reasons to believe that people's posterior judgments are affected by sampling regime in such non-normative ways. For example, in a classic experiment, Griffin and Tversky (1992) had participants give confidence estimates about whether a series of coin flips came from a coin that had a $2 / 3$ bias towards heads or one that had a $2 / 3$ bias towards tails. By systematically varying both the strength of the evidence (that is, the proportion of heads/tails in the sample) and the weight of the evidence (the number of coin flips), they found that participants based their confi- 
dence judgments disproportionately on strength. Thus, they were overconfident about small samples with high strength (heavily leaning towards one of the two hypotheses) and underconfident about large sample with moderate strength. Recently, Kvam and Pleskac (2016) found a similar tendency to over-emphasize the strength of evidence compared to its weight. In a perceptual judgment task they had participants make binary choices and give confidence estimates about the number of red and green dots on a screen, while varying the total number of dots. Like Griffin and Tversky (1992), they found that participants were more influenced by strength (proportion of red/green dots) than weight (total number of dots). This was true both in terms of their choices (choices were always between two generating assumptions about the number of red/green dots) and their confidence judgments. Importantly, they also found that participants were not impaired in recalling the total number of dots on the screen, that is, their overemphasis on strength could not be explained by the inability to perceive differences in weight.

\section{Experiment 1}

Experiment 1 manipulated sampling goals in a simple repeated Bernoulli sampling task that shared all the characteristics of the task described in the modeling section. The sampling goal was manipulated between participants who either had to estimate the overall value of $\theta$ (estimation condition) or decide whether $\theta$ was greater or lower than 0.5 (binary condition).

We predicted that sampling goals would affect both the number of samples collected on average (higher for the estimation vs. binary goal), and the relationship between stopping points and current sample composition. As outlined in the previous section, we expected participants engaged in sampling with the binary decision goal would be more likely to stop early when strong evidence is encountered (average closer to 0 or 1 ) and more likely to continue when evidence is mixed (average closer to 0.5 ).

\section{Methods}

Participants. 276 participants were recruited via Amazon Mechanical Turk. They were paid $\$ 2$ for participating with an option to win a bonus of up to another $\$ 2$ (explained below).

Stimuli. Participants were told that they were repeatedly drawing cards from 50 card decks consisting of 200 cards each. Cards could be either red or blue, and the distribution of red and blue cards in each deck was determined semiuniformly (using an evenly-spaced distribution of Bernoulli probabilities $\theta$ that were then used to randomly draw the cards for each deck). Participants were explicitly told to assume a uniform distribution of numbers of red and blue cards in each deck, as well as being told that the order of cards in each deck was completely random ("well shuffled").

Procedure. A schematic of the procedure is shown in Figure 3. For each card deck, participants could repeatedly (up to 24 times) turn over cards using a button press to reveal their color. A counter on the screen would tell how much of potential bonus remained after each sample (which cost $\$ 0.05)$. For each card deck, the potential bonus started at $\$ 2$. At any point participants could also decide to move on to the secondary task (binary choice or estimation) via a different button. In the binary task, participants would then make a two alternative forced choice decision of whether they thought there were more red or more blue cards in the deck. In the estimation task they gave an exact estimate using a sliding bar whose handle had two lines that indicated the .2 interval within which their response would be counted as correct. After giving their estimate, all 200 cards of the card deck were revealed and participants received feedback on their choice or estimate. If their estimate was correct, their potential bonus was recorded as the bonus that remained after the sampling phase, otherwise the it was recorded as $\$ 0$. At the end of the task, one card deck was chosen at random and participants were actually paid the bonus earned on that deck. Participants were aware of the rules of the game and answered some comprehension questions to ensure they had read and processed the instructions.

\section{Results}

To recap, our two main predictions motivated by the modeling were that participants in the estimation condition would collect larger samples on average, and that participants in the binary condition would base their decision when to stop on the current strength of the sample.

Sample size. Figure 4A shows the distribution of sample sizes by condition. The vertical line in each plot corresponds to the average number of samples the optimal stopping model would take when sampling from the same underlying distribution with the same goal, sampling cost, and final reward as participants in the experiment (assuming very minimal decision noise, $\tau=0.01$ in Equation 6). In line with the model predictions (see also Figure 2A), participants in the estimation group took larger samples on average than participants in the binary group $(t(211)=-11.18, p<0.01)$. This suggests that people were aware of the trade-off between sampling cost and accuracy and were willing to incur higher cost (more samples) in the more difficult task. There were also visible spikes for certain sample sizes $(8,10$, and 12$)$ in the estimation condition, suggesting that these were particularly salient stopping points for some participants (cards on the screen were aligned in columns of four, so spikes at 8 and 12 may be the result of an aesthetically inspired fixed-samplesize stopping rule). 
1. Sampling phase

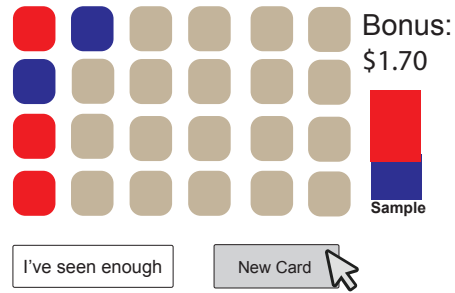

2. Post-sampling Response

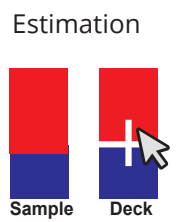

\section{Binary}

Are there more red or blue cards in the deck?

${ }^{\text {Red }}$

\section{Feedback}

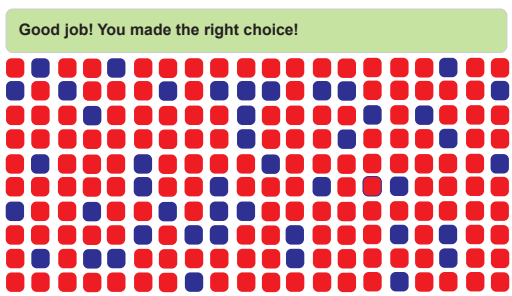

Figure 3. Task used in Experiment 1. (1) In the sampling phase, participants were sequentially turning over cards from a card deck. On every trial, they had to decide to turn over another card, or proceed to give their response. The current number of red or blue cards and their remaining bonus were shown on the screen. (2) After sampling, participants gave either an estimation or binary response. Participants in the estimation group had to drag a slider on a vertical bar representing the number of red or blue cards they believed to be in the entire card deck ( 200 cards). Participants in the binary group indicated if they believed there were more red or blue cards in the deck. (3) After every trial (card deck), participants received feedback about the true number of red and blue cards in the deck.
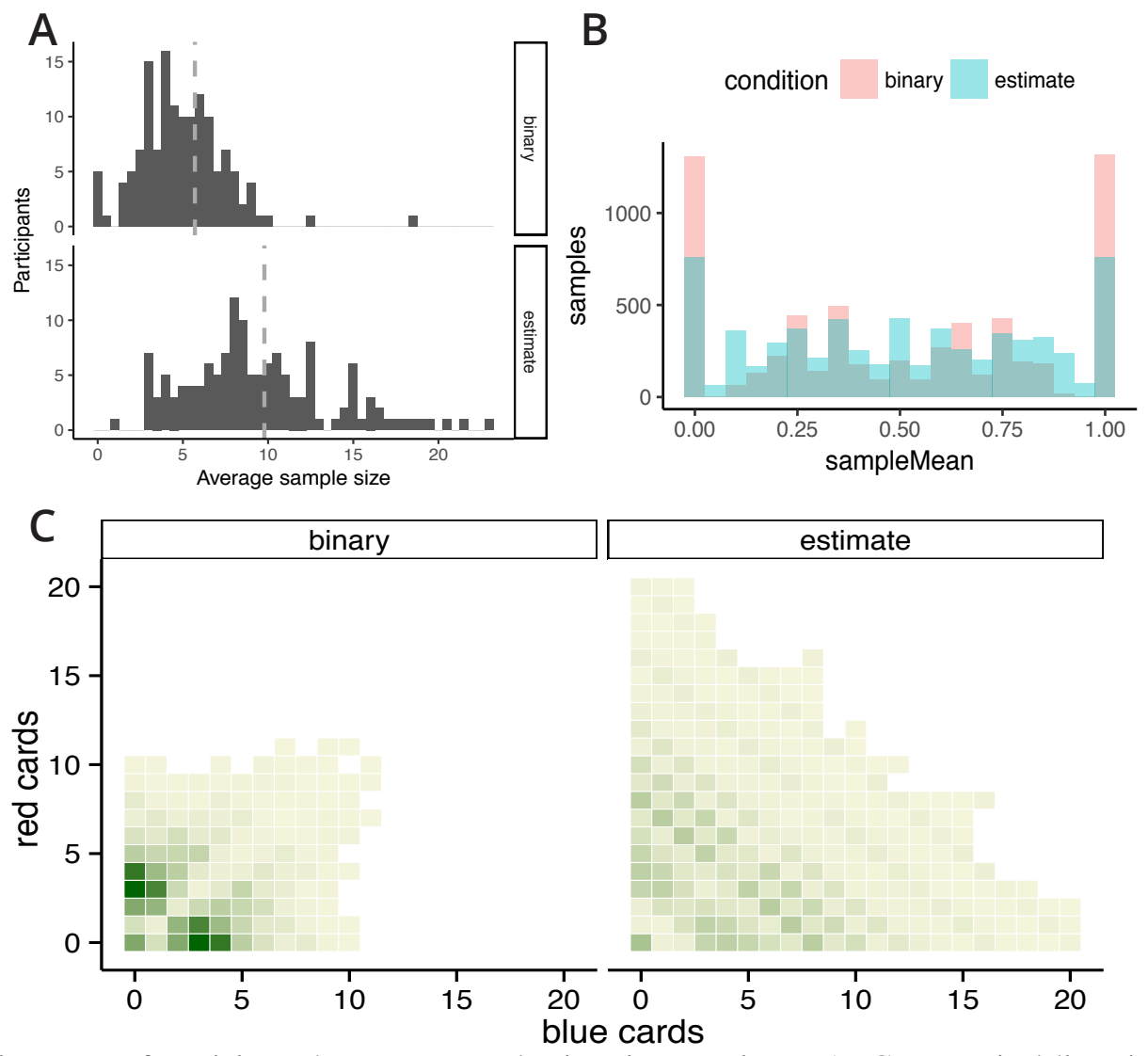

Figure 4. (A) Histogram of participants' average sample sizes in Experiment 1. Grey vertical lines indicate the expected average from the model run on the same distribution that generated experiment data. (B) Distribution of sample averages in Experiment 1. (C) Stopping probabilities (dark means high) by sampling goal. 
Note that, compared to the expected average sample size of the low-noise model, participants did not appear to systematically over- or undersample. That is, the data in Figure $4 \mathrm{~A}$ fall roughly around the vertical line that indicates the model predictions. However, this result should be treated with caution, because the expected sample size predicted by the model heavily depends on the value of the decision noise parameter, $\tau$. As $\tau$ increases (noisier decisions), the model becomes more likely to stop earlier, which overall decreases the expected sample size. Thus, while behavior seems roughly in line with a low- $\tau$ model, if people were acting it is possible that people actually oversample if their true decision rule is noisier than assumed here.

Stopping points. Figure 4C shows the proportion of times participants collected a sample of a given number of red and blue cards (dark green indicates that more participants collected that kind of sample). There is a clear difference in the stopping patterns of the two groups. Participants in the binary group were more likely to stop in early extreme states (mostly red or mostly blue cards), but continued sampling if early evidence was mixed. Participants in the estimation group, on the other hand, showed no discernible stopping pattern based on current sample composition. For a given sample size, they exhibited a fairly equal stopping probability across different proportions of red/blue cards. The above-mentioned preference for certain salient sample sizes $(8,10$, and 12) in the estimation group is reflected by the diagonal "ridges" in the stopping matrix.

Sample Composition. Another way to quantify these stopping strategies is to analyze the characteristics of the sample that participants collected. Figure 5 shows the average strength of each sample by sample size and condition. Strength is defined as the proportion of data points with the more common outcome (red or blue) in a given sample. High strength thus indicates a sample average near 0 or 1 and low strength corresponds to an average near 0.5. Compared to the estimation group, the binary group exhibits a stronger negative relationship between strength and sample size, which arises because participants were more likely to stop upon encountering high-strength evidence early on in a trial. Thus, there exist a significant interaction between strength and condition in their effect on final sample size. This inference was based on a model comparison between two mixed effects regressions (Barr, Levy, Scheepers, \& Tily, 2013) of final sample size. The null model included fixed effects of sample strength and condition, as well as random intercepts for subjects. The augmented model had an additional fixed effect for the interaction between strength and condition. A likelihood ratio test revealed a significant improvement in model fit $\left(\chi_{L R}^{2}(1)=534, p<0.001\right)$.

Finally, we also examined the distribution of sample averages resulting from these stopping rule differences. Based on the model, we expected that the binary group would be more

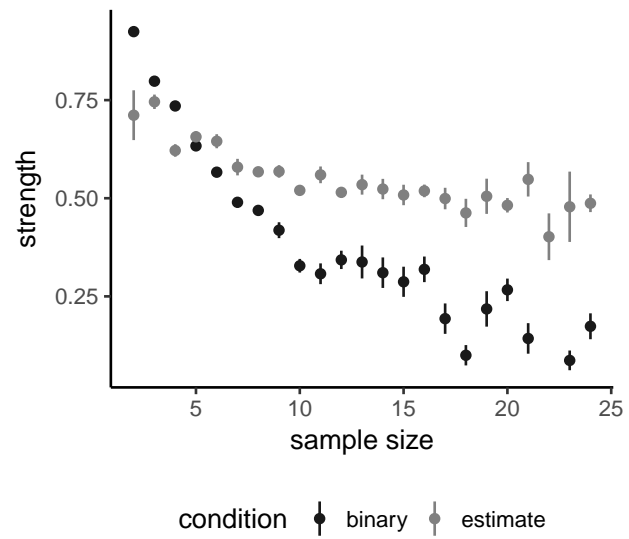

Figure 5. Sample strength - proportion of data points with the more likely outcome (red or blue)— by sample size and condition.

likely to end up with samples of high strength (averages near 0 or 1). Figure $4 \mathrm{~B}$ shows the distribution of sample averages by condition. Indeed, the group with the binary goal showed a much larger proportion of samples with extreme averages (all red/blue) compared to the estimation condition. As a consequence the average sample strength of participants in the binary group was significantly higher than for participants in the estimate group $\left(M_{\text {binary }}=0.66, M_{\text {estimate }}=.57\right.$; $t(274)=10.85, p<0.001)$.

Confidence. The main purpose of this experiment was to test if participants would adjust their stopping regimes in line with their goals. However, since participants gave confidence ratings after giving their response, we were also able to start asking how certain they were about their inferences about those samples.

Based on prior work (e.g., Griffin \& Tversky, 1992; Kvam \& Pleskac, 2016), we expected that confidence ratings about binary choices would be influenced more by the strength of the evidence (proportion of data points with the more common outcome) than the weight of the evidence (sample size). We therefore investigated how these two factors influenced confidence ratings of participants in the binary group (there is no straightforward way to conduct this analysis for participants in the estimation group, because evidence strength for the estimation goal does not translate to extremeness of the outcomes).

For every trial, we first regressed participants' individually z-scored confidence ratings against the true probability of making a correct choice, computed on the basis of the data in the sample (number of red and blue cards), assuming a flat prior to start with $(\operatorname{beta}(1,1))$. The residuals of this regression were then used to investigate the effect of strength and sample size. Figure 6 shows the average residual confidence rating by strength (left) and sample 

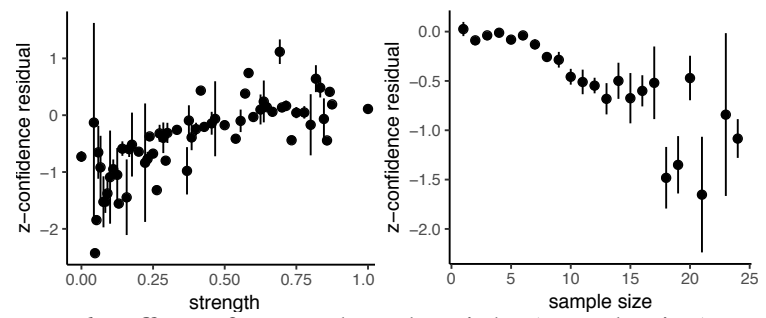

Figure 6. Effect of strength and weight (sample size) on average confidence residuals after accounting for the true probability of making a correct choice. Data is from the binary group only, because the influence of strength and weight cannot be separated for the estimation group. Error bars represent standard errors.

size (right). In line with our predictions, we found a significant positive correlation of residual confidence with strength, $r(6426)=0.28, p<0.001$, and a negative one with sample size, $r(6426)=-17.4, p<0.001$. This confirms that participants were more confident about small samples with high strength than large samples of low strength, even after accounting for the true probability about of making a correct choice (i.e., after accounting for what their confidence level should be according to an optimal Bayesian learner).

\section{Summary}

Experiment 1 tested whether two inference goals, a binary and an estimation goal, would differentially affect people's strategies for choosing when to terminate search. We found that participants made stopping decisions in a manner that is broadly consistent with the optimal stopping analysis presented in the first part of this paper. Participants in the binary condition decided to stop earlier when initial evidence indicated a sample average near 0 and 1 , but continued sampling if the sample average was more mixed (i.e., near 0.5). Participants in the estimation group, on the other hand, showed no such pattern and were instead more likely to collect samples of a similar size on each trial. Although this is not exactly the behavior the optimal learner model predicts, it leads to a qualitative difference in the distributional characteristics of samples collected by both groups. Binary samplers ended up with more small samples with high strength (averages near 0 or 1) and larger samples with moderate strength, whereas estimation samplers showed a much weaker relationship between strength and sample size.

Furthermore, an analysis of confidence ratings revealed that binary samplers were more confident about choices that were based on small samples with high strength compared those based on large samples with moderate strength. Importantly, this analysis was conducted after accounting for the true Bayesian probability of making a correct choice. This confirms that participants exhibited the same tendency to discount the effect of sample size that has been found in prior work on probability estimation (Griffin \& Tversky, 1992; Kvam \& Pleskac, 2016; Tversky \& Kahneman, 1971).

\section{Experiment 2}

Experiment 1 established that people adapt their stopping rule to different sampling goals in a way that is broadly consistent with the predictions of the optimal analysis reported above. One goal of the second experiment was to test if these stopping regime differences can be replicated in a sampling task with continuous rather than binary outcomes. To this end, we changed the experimental paradigm to involve normally distributed outcomes (the optimal model predictions are similar for this case, see Appendix).

The second goal of Experiment 2 was to investigate how differences in stopping regimes affect subsequent generalization about the population learners sample from. For example, consider a job recruiter who is repeatedly testing potential employees to determine their suitability for a position. If she has a binary goal (e.g., "is this candidate above or below average?"), she may stop interviewing candidates early on if they perform either very well or very poorly (and advance the former and reject the latter). With an estimation goal ("what's the suitability of this candidate?') she might aim for the same number of interactions with each candidate. In either case, she will develop beliefs about the suitability of each candidate over time and also form an impression about the distribution of suitability across an entire pool of candidates.

Here, we investigate how sampling strategies affects such population beliefs. Specifically, we ask if sampling with a binary goal leads to an increased belief in the likelihood of extreme cases (e.g., very poor or or very qualified candidates). This is because, as shown in the previous experiment and the modeling results, sampling with a binary goal results in more small samples with extreme averages when compared to estimation sampling. If learners do not take into account that these extreme samples are often small (e.g., few interactions with a candidate) and downweigh their importance, they might end up believing that the underlying distribution has more extreme values than is actually the case. Returning to the recruiter example, if she uses a binary stopping strategy and does not account for differences in sample size, she might end up believing that the world holds more candidates that are either very talented or very ill-suited. Note that we have reason to believe this insufficient regard to sample size will occur based on numerous findings in the psychology of reasoning (e.g., Griffin \& Tversky, 1992; Kvam \& Pleskac, 2016; Kahneman \& Tversky, 1972; Kareev, Arnon, \& Horwitz-Zeliger, 2002). The confidence data from Experiment 1 also confirmed this by showing that binary samplers placed relatively more weight on smaller samples than larger 
ones.

To test the effect of sampling strategies on beliefs, Experiment 2 asked participants to give explicit posterior estimates about the underlying distribution of the samples they collected. Changing the experimental paradigm to continuous outcomes facilitated this measurement of people's population beliefs, because it meant that distributional characteristics (e.g. averages) were in the same units as the sampled outcomes, so that they did not need to translate discrete values (e.g., red and blue cards) to probabilities and averages.

\section{Methods}

Participants. 100 participants were recruited via Amazon Mechanical Turk. They were paid $\$ 1.50$ for participating with an option to win a bonus of up to another $\$ 1.50$ (explained below).

Trial Structure. Participants' were told they worked as a "lake biologist" who had to test a total of 70 lakes to find out about each lake's algae concentration. On each trial of the experiment, that is for each lake that was tested, participants could repeatedly order water tests from the laboratory. Test results ranged between -50 (low algae concentration) and +50 (high algae concentration). All test results were drawn from one of two normal distributions with different means $(+10$ or -10$)$ and a fixed standard deviation of $12^{3}$. The two distributions (with means of +10 or -10 ) corresponded to two types of lakes that participants tested, each of which was associated with a different "chemical" (see below). Every test result was displayed by a dot on a horizontal scale (see left panel of Figure 7), which remained on the screen for the entire trial so participants could see all test results for a given lake. Participants had to draw at least one test result per lake, but could collect up to 20 if they desired. Each test score cost $\$ 0.10$ that was deducted from a potential starting bonus of $\$ 1.00$ (see below for details on the bonus payment). The current value of the remaining bonus was displayed on the screen throughout each trial. After participants decided they had collected enough test scores for a given lake, they were asked to make an assessment of that lake, which differed between conditions.

Participants were randomly assigned to one of two sampling goal conditions. Those with a binary sampling goal had to decide, for each lake, if its true algae score was above or below 0 . Participants with an estimation goal had to estimate the true algae score directly, by moving a vertical sliding bar over the horizontal scale. Participants' responses were then compared to the underlying mean that generated the test scores for the given lake (10 or -10). In the estimation condition, a response was counted as correct if it was within 10 points of the true underlying mean. Participants received no feedback about their response.
Instructions. Participants were told to pretend they were working as a lake biologist to examine the "algae score" of a series of lakes. They were told that algae scores could be assessed by repeatedly conducting "noisy" water tests, the mean of which would eventually converge to a lake's true algae score if enough tests were conducted. To illustrate that tests were noisy, participants were shown a distribution of test results for four lakes with different true algae scores. The data in these examples corresponded approximately to the true standard deviation of the data (12). Participants were then introduced to their respective sampling goal (binary and estimate) and given a quiz about the task that they had to pass before being allowed to move on to the next part of the experiment.

Sampling phase. First, in a training phase, participants took samples from 20 lakes to familiarize themselves with the task. The mean of each training lake was drawn from a uniform distribution between -35 and +35 . Participants received feedback on their assessments, that is, they were told if their choice (binary condition) or estimate (estimation condition) was counted as correct. To allow them to explore the task, these first 20 lakes were exempt from being chosen for the final bonus calculation (see below).

After the training phase, participants were told that the remaining 50 lakes that needed testing had been treated by one of two chemicals, chemical A or chemical B, which were blue or orange. Participants were also told that researchers expected the chemicals to have different effects on the algae concentration of a lake. In addition to their existing task (that is, their binary or estimation goals) participants were instructed to pay attention to any effect of the chemicals and learned that they could win a "surprise bonus" at the end, if they did well on a final evaluation of the impact of the chemicals. For the remaining 50 trials, participants alternated between testing lakes treated by each chemical. Which chemical (blue or orange) was the high and low one, was counterbalanced between participants.

Final Evaluation. After testing 25 lakes treated by each chemical, participants completed a final "evaluation task", which was meant to elicit their beliefs about the impact of the chemicals. For each chemical (order was counterbalanced between participants), they were asked to estimate (i) the average algae score of lakes treated by that chemical, (ii) the median algae score of lakes treated by the chemical, and (iii) the algae score at the 25 th and 75 th percentiles of the distribution of lakes treated by each chemical. All estimates were made by dragging vertical sliders on a horizontal scale ranging from -50 to +50 . To make estimates of the $25 \mathrm{~h}$ and 75 th percentile, the prior estimate of the median remained on

\footnotetext{
${ }^{3}$ The choice of standard deviation was made based on model simulations that maximized the difference in the effect of the binary and estimation stopping goals on the resulting distribution of sample means.
} 
1. Sampling

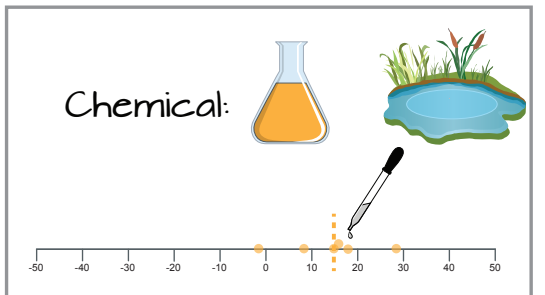

2. Response

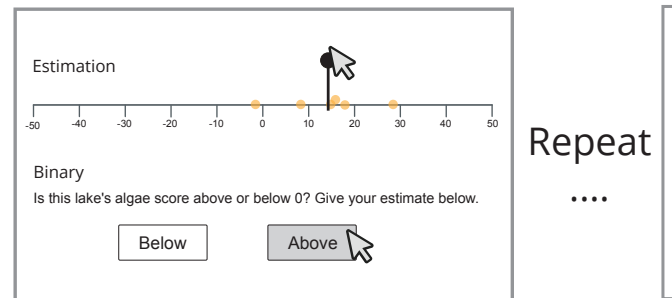

3. Posterior Estimates

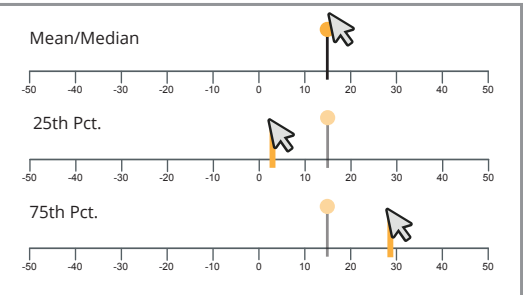

Figure 7. Task used in Experiment 2. This example shows trials involving the orange chemical, which was alternated with the blue chemical. (1) In the sampling phase, participants repeatedly received test results about a lake's algae score and had to decide when to stop ordering more results (their remaining bonus was always displayed on the side of the screen and is not shown here). Test results were displayed as dots on a scale between -50 and 50 and the current average was always indicated by a dotted vertical line. (1) After sampling, participants either gave an estimation or binary response. The estimation group had to drag a vertical line to their best estimate of the lake's true algae score. Participants in the binary group had to indicate if they thought the lake's true algae score was above or below zero. (3) In the posterior judgment phase, participant gave four estimates (mean, median, 25th percentile, 75th percentile) about the overall population of algae scores of lakes treated by each of the chemicals. (All posterior estimates were made on separate consecutive screens).

the screen as a vertical line and additional sliders appeared on either side for the two percentile estimates (see rightmost panel of Figure 7).

While the mean was elicited by directly asking participant about the average algae score per chemical, the median and percentile estimates were elicited via a bisection method. This method asked participants to successively subdivide their subjective cumulative distributions of the lakes' algae scores (for examples of the bisection method for eliciting subjective credible intervals, see e.g., Winkler, 1967; Garthwaite, Kadane, \& O'Hagan, 2005; Murphy \& Winkler, 1977; Peterson, Snapper, \& Murphy, 1972). For example, to estimate the median, participants were told to "...(think) about the lakes treated with the blue/orange chemical. Can you identify a value on the scale such that the scores of half of the lakes would fall below that value and the other half above?". Similarly, the 25th percentile was then elicited in the following way: "Now, think about only those lakes with a score that falls below the value you just identified. Can you now determine a new value such that the scores of half of these lakes (so a quarter of the total) lie below that value?".

Bonus. To determine a participant's final bonus, we randomly selected one of the lakes they tested during the main phase of the experiment. If their assessment of that lake was correct, they received the remaining bonus from that trial (that is, $\$ 1.00$ minus $\$ 0.10$ per water sample they collected). For the final evaluation, participants received an additional bonus of $\$ 0.50$ if the difference in their mean estimates of the two sets of chemicals (with true means of -10 and +10 ) was within 10 points of the true value (the true difference was 20).

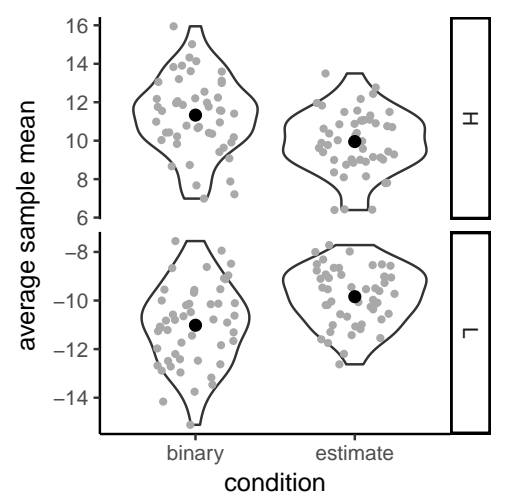

Figure 8 . Average sample mean by condition and generating process.

\section{Results}

Sampling. Figure 9A shows the proportion of times participants stopped requesting new test results for any given lake, given the current average of test scores and the number of test scores collected. Visual inspection of this plot alone reveals differences in the distribution of stopping points between the two conditions. Figure 9B shows the corresponding model predictions (for model details, see Appendix).

Stopping points of participants in the estimation group fall symmetrically around the true generating mean of the high $\left(\mu_{h}=10\right)$ and low $\left(\mu_{l}=-10\right)$ distribution, whereas stopping points in the binary condition reveal longer "tails" in the direction away from the binary criterion (0). This pattern emerged because participants in the binary group terminate search early upon encountering far-from-criterion evidence, 


\section{A. Data}

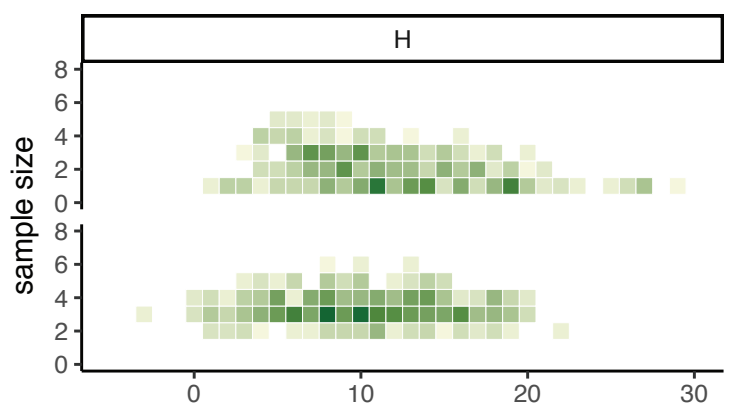

B. Model

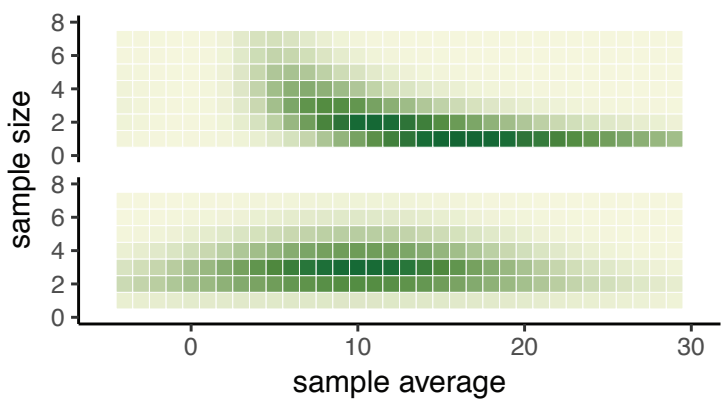

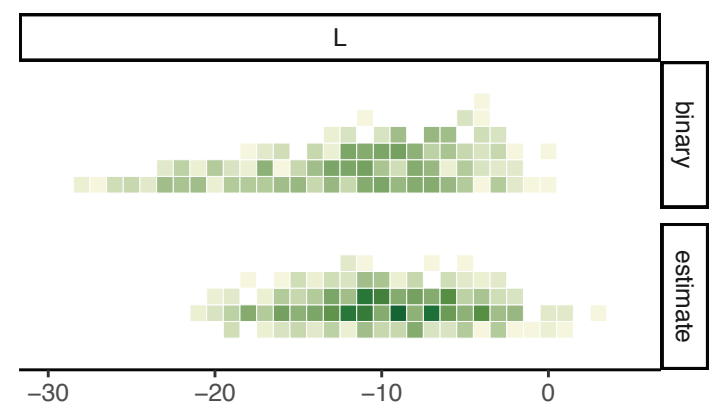

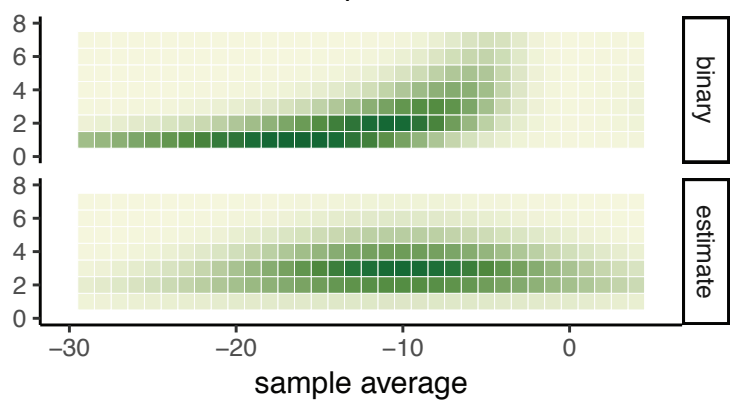

Figure 9. (A) Proportion of times (dark means high) participants terminated sampling given the current sample average and current sample size in Experiment 2. Data are shown separately by sampling goal and by generating process (samples either had High (10) or Low (-10) underlying mean and standard deviation 12. (B) Optimal stopping predictions given the same generating process as used in Experiment 2 (see Appendix).

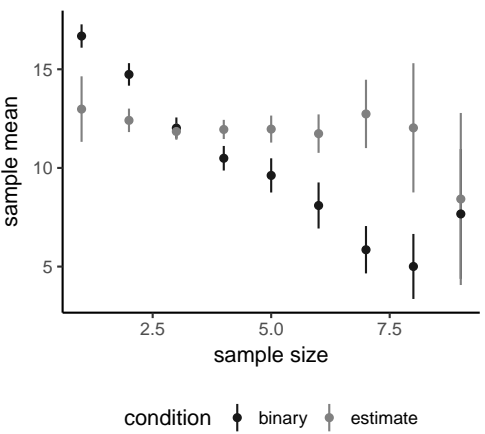

Figure 10. Relationship between sample size and sample average, by stopping goal in Experiment 2. Data include both the training phase and the main phase of the experiment (but each of these individually reveal a similar pattern).

and otherwise continue.

Sample characteristics. To further quantify the relationship between stopping behavior and sample content, Figure 10 shows how the absolute value of a given sample average (i.e. its deviation from 0 ) varies with its size (i.e. the number of test scores drawn). In the binary group, the two are negatively related, revealing that participants terminated search earlier when the sample average was far away from 0 (the binary criterion), and were more likely to collect more information when the average was closer to 0 . Thus, there exist an interaction between absolute sample mean and condition in their effect on final sample size. We verified this relationship based on a model comparison between two mixed effects regressions of final sample size. The null model included fixed effects of the absolute sample mean and condition, as well as random intercepts for subjects. The augmented model had an additional fixed effect for the interaction between absolute sample mean and condition. A likelihood ratio test revealed a significant improvement in model fit $\left(\chi_{L R}^{2}(1)=182, p<0.001\right)$.

Figure 8 shows the difference in average sample means between lakes treated with the high and the low chemical. The dotted gray line is the expected true difference assuming equal sample size (i.e. 20, given the two chemical's means if -10 and 10). As expected, the estimation condition yields a distribution of sample averages around the expected value ( $M=19.8$ ). Data generated by binary samplers, on the other hand, yields a larger difference between the average sample means of the two datasets $(M=22.23, \mathrm{t}(98)=5.82$, $\mathrm{p}<0.001)$.

Posterior beliefs. Figure 11 shows the posterior estimates participants gave for the mean, median, upper (75th) and lower (25th) percentile of the two sets of lakes (high and low value). Across all measures, the posterior estimates from 


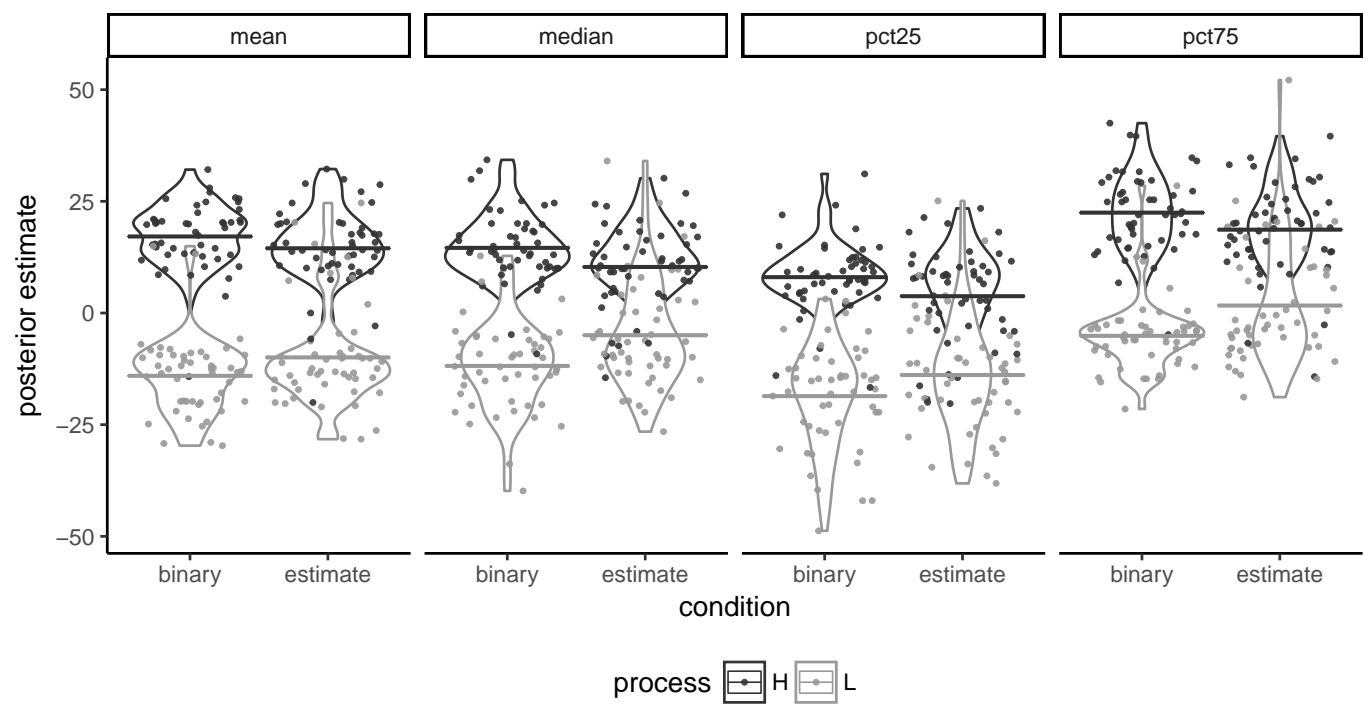

Figure 11. Experiment 2: Posterior estimates for High and Low process, by stopping goal.

the binary group were more extreme (higher for the high and lower for the low value lakes) than those in the estimation group. To analyze the data further, we conducted four separate analyses of variance, one for each posterior measure. To do so, we flipped the sign of estimates of the low value lakes and included a predictor for lake type (high or low) in the analysis to control for potential effects of scale usage (generally, participants made less extreme estimates for the low value lakes, see Figure 11). Note that combining data from the low and high value lakes meant that the 25th and 75 th percentile estimates were transformed into measures of the outer interval (75th for the High, and 25th for the Low value lakes) and the inner interval (the reverse). Controlling for lake type, we found a significant main effect of condition on all four posterior measures $\left(F_{\text {mean }}(197)=6.68, p=0.01\right.$, $F_{\text {med }}(197)=15.79, p<0.001 ; F_{\text {outer }}(197)=7.45, p=$ $\left.0.006 ; F_{\text {inner }}(197)=14.59, p<0.001\right)$.

Although this experiment revealed robust differences in posterior estimates, it is possible that this was caused by other factors besides differences in sample composition. For instance, participants in the binary group might have formed more separated perceptions of the algae score distributions of the two lake types simply because they were giving binary responses throughout the experiment. To check whether sample composition alone affected posterior estimates, we correlated the difference in participants' posterior estimates for the high and low value lakes with the uncorrected mean difference of the two lake types that each participant experienced. We found a significant positive correlation for all difference measures, except the mean $\left(r_{\text {mean }}=0.13, p=0.19 ; r_{\text {median }}=\right.$ $0.24, p=0.015 ; r_{\text {higher }}=0.27, p=0.008 ; r_{\text {lower }}=0.21, p=$ 0.032 ). This strongly suggests that the difference in posterior estimates of participants in the two conditions was at least partly driven by the differences in the data that they saw, and thus was not just an effect of response mode.

\section{Summary}

This experiment showed that manipulating the sampling goal (binary vs. estimation) had a similar effect on people's stopping rule in a Gaussian sampling task, as it had in the Bernoulli task from Experiment 1. Like our optimal stopping analysis predicts, participants in the binary group terminated search earlier when their current sample average was far from their decision boundary. This again affected the distributional characteristics of the samples that participants collected. Participants with a binary goal ended up with more samples with particularly high or particularly low sample means. Dividing these samples across two generating processes (with a high or low mean) meant that the average difference between those processes was exaggerated for those participants.

Our main question was whether these stopping rule induced differences in sample composition would affect people's posterior estimates about the generating distributions. This question was motivated by prior work that has shown that people pay more attention to the strength of evidence than its weight. We therefore expected that the data collected binary samplers might affect their population-level inferences, unless they were able to counteract this bias. Indeed, we found that participants with a binary goal gave more extreme estimates of the distributional parameters. This effect was stronger for participants whose sampling regime resulted in greater deviation of the average sample means from the true generating distribution. This indicates that the group-level difference in posterior estimates was not just an effect of the response mode, but was directly related to the 
imbalance of samples collected by participants in the binary group. ${ }^{4}$

\section{General Discussion}

We started this paper by noting that there are (at least) two ways in which learners can exert control over how they sample information from their environment. Being able to select what to sample is often associated with the potential for sampling bias, because it allows learners to collect samples that do not preserve the properties of the underlying distribution (Fiedler, 2000). In this paper we argued that, even when learners cannot select particular kinds of information, the mere decision of when to stop sampling for information can impact samples in a way that has the potential to bias people's beliefs about the world. We show that this can happen under two conditions. First, the potential for bias occurs when learners collect samples with the goal of making a binary choice (e.g., "is this coin biased towards heads or tails?"). This leads them stop particularly early when evidence is strong (e.g., all heads or all tails) and later when evidence is mixed, resulting in a negative correlation between strength and sample size. The second condition leading to sampling bias is that, when learners give estimates about the distribution that generated samples, they fail to sufficiently account for sample size and thus give more weight than they should to small, extreme samples.

In the remainder of this paper, we will discuss how these results contribute to our understanding of the relationship between sampling and belief formation, and describe how our findings relate to prior work on stopping behavior more broadly.

\section{Insensitivity to sample size as a source of sampling bias}

Prior work on selective sampling suggests that people find it difficult to account and correct for the process under which samples were generated (Fiedler, 2008, 2000). Here, we found another example of such sampling-related "metacognitive myopia". Specifically, we show that people's insensitivity to sample size, which has been found in a variety of other tasks (e.g., Griffin \& Tversky, 1992; Kvam \& Pleskac, 2016; Kahneman \& Tversky, 1972; Kareev et al., 2002), can be a source of bias when sample size is correlated with sample strength (as was the case for samplers with a binary goal).

Note that correlations between evidence strength and evidence weight naturally occur in many kinds of sampling processes based on binary decisions. For example, the same relationship emerges from evidence accumulation models in a variety of psychological tasks, such as value-based choice (e.g. Pleskac, Busemeyer, \& Others, 2010; Goldstein \& Gigerenzer, 2002), recognition memory (Ratcliff, 1978), categorization (e.g. Nosofsky \& Palmeri, 1997), and perceptual decision-making (e.g. Bogacz et al., 2006; Usher $\&$ McClelland, 2001). These models often rely on a binary decision criterion and thus accumulate more information (measured as reaction time) when evidence is mixed and less information when it is strong.

There is also reason to believe that such a relationship could emerge in other real-world scenarios, in which people collect information based on binary goals. For example, consumers often seek information to decide if they would like to purchase or recommend a product or not, social media users are now mostly given the options of a "thumbs up" or "thumbs down" rating of new content, judges need to seek evidence in order to find a defendant guilty or not guilty, and intelligence analysts have to process incoming data to decide whether some new activity poses a threat or not. Furthermore, many activities we observe are inherently adhering to a criterion-based (binary) stopping rule. For example, certain kinds of competitions end earlier when there exists a dominant competitor, but take longer if strength is more evenly distributed.

Given that binary sampling goals are so ubiquitous, it is likely that binary stopping rules and the resulting effects on sample composition occur in many situations outside of the lab. In future work, we therefore hope to address the consequences of binary criterion sampling in such real-world scenarios, and investigate if it can bias people's judgments in a similar way as demonstrated here.

\section{Conclusions}

The present paper examines how the decision to stop collecting information can potentially bias judgement and choice. To summarize, we first showed that alternative sampling goals (either binary choice and parameter estimation) differentially affected people's strategies of when to stop sampling. These differences induced differences in the distribution of statistical evidence that the learners encountered. Such differences are not problematic assuming that the learner performs the proper correction (specifically a sample size correction to the mean). However, in the second study

\footnotetext{
${ }^{4}$ In a follow-up experiment, we further tested how the response mode (binary choice or continuous estimate) affects posterior estimates in addition to, or interaction with, the impact of sample characteristics. Groups of participants were either assigned to respond with a binary choice or a continuous estimate and presented with data that was either generated by the optimal model run with a binary goal condition or with an estimation goal $(2 \times 2$ design). The task was identical to Experiment 2, except that participants were told when to stop sampling each lake.

We found that sample characteristics (binary data vs. estimation data) affected posterior estimates in the same direction as Experiment 2 even when the response mode was the same. However, we also found that response mode had a significant effect on one of the posterior measures irrespective of the data. Specifically, estimates of the inner confidence bound (the lower tail of the high-value lakes and upper tail for low-value lakes) were further away from zero (the binary criterion) for participants with a binary response mode.
} 
we found that people had biased estimates of the population process because they fail to correct for the bias their decision to stop sampling introduced. Perhaps most importantly, the analysis presented here shows that even natural sampling can lead to biased inference. The results and analysis also parallel recent concerns in the statistical literature about the dangers of terminating data collection for research studies based on the strength of early samples (Simmons, Nelson, \& Simonsohn, 2011).

\section{Appendix}

\section{Optimal Stopping: Gaussian sampling}

To demonstrate the impact of stopping on sample composition, consider a task in which learners draws independent data points $x$ from a normally distributed random variable $X$, with unknown mean and known variance $\sigma^{2}$.

Assume that the learner incurs a small cost $c$ for every draw of the distribution. Let's also assume that the learner will subsequently have to answer one of the following two questions (sampling goals).

1. Binary: Find out if the mean is greater above or below 0 .

\section{Estimation: Estimate the mean.}

Each goal is associated with a reward that is a function of the true value and the participant's estimate. The learner's task is to decide when to stop sampling and provide their estimate given this cost function, the current sample, and the sampling cost. Assuming that there exists a maximum number of draws that learners are allowed to take this can be framed as an finite-horizon optimal stopping problem. At every state (defined by the size of the current sample, $n$, and the current sample average $\bar{x}$ ), an optimal decision maker should compare the expected value of stopping and of continuing and choose whichever is higher. Thus the expected value of a state, given a horizon of a maximum of $T$ flips is

$$
V_{t}^{(T)}\left(t, \bar{x}_{t}\right)=\max \left\{V_{\text {stop }}, V_{\text {cont }}\right\}
$$

where

$$
V_{\text {stop }}=u_{\text {stop }}\left(t, \bar{x}_{t}\right)-t c
$$

and

$$
V_{\text {cont }}=E\left[V_{t+1}^{(T)}\left(t+1, \bar{x}_{t+1}\right)\right]
$$

and where $u_{\text {stop }}($.$) is the expected utility of the post-$ sampling task, which depends on a learner's sampling goal (see below). The value of continuing to obtain another sample is a learner's expectation over possible future states given their current knowledge. The probabilities in the expectation are based on a beta distribution parameterized by the outcomes observed so far. The value of the final state (when $n=T$ ) is just the expected value of stopping, which, along with the Markov property, means that this problem is solvable by backwards induction (Ferguson, n.d.).

A learner's posterior belief about the value of the mean is $\theta \sim N\left(\mu, \sigma^{2}\right)$. The hyperparameters of this distribution, $\mu$ and $\tau$, are inferred conditional on the data and a learner's prior belief.

The utility of stopping under the binary goal is

$$
u_{\text {stop }}^{\text {bin }}=\max \left\{1-\Phi_{0}(\theta, \tau), \Phi_{0}(\theta, \tau)\right\} r,
$$

where $r$ is the reward for making a correct choice.

The expected utility from stopping under the estimation goal requires specifying a cost function over the distance between the participant's estimate and $\theta$. For simplicity's sake, assume that the learner's answer counts as correct whenever their response lies within a .2 interval surrounding the true value, and incorrect otherwise. The expected utility from stopping under this goal is

$$
u_{\text {stop }}^{\text {est }}=\left[\Phi_{\theta-.1, \theta+.1}(\theta, \tau)\right] r,
$$

where $\mu$ and $\tau$ are the posterior hyperparameters of the distribution of $\theta$.

Predictions. To predict behavior using this model, we used the following parameter values: $c=\$ 0.05, r=\$ 1.0$, $T=20$. We assumed that the learner knew the standard deviation $\sigma=12$. Besides sampling cost, these were the same values used in Experiment 2. Sampling cost was doubled in the experiment because piloting revealed that participants took much larger samples than the model predicted. To generate predictions, we used a softmax rule from Equation (6) with $\tau=.05$. Again, these values were chosen for illustrative purposes and many qualitative results hold across a broader range of values.

Stopping. Figure 12A shows the model's predictions for the learner's decisions to stop (white) or continue (green) given the current state (sample average and sample size). Under the binary goal, learners should continue sampling if the current sample average is closer to 0 (the binary criterion). In contrast, the estimation goal predicts a stopping rule that only depends on sample size and is independent of sample composition.

Based on the stopping matrix in Figure 12A we derived expected probabilities for the samples that learners eventually end up with. Figure 12B shows the likelihood of different stopping points assuming a true underlying mean $\mu=10$. Again, binary learners end up collecting smaller samples with averages far from 0 or larger samples with averages closer to 0 . Given the fixed-n stopping rule of the estimation condition, the sample averages corresponds to the true sampling distribution of the mean, $\mu=10$.

Figure 13 shows the resulting relationship between the sample average and sample size. As was the case in the Bernoulli example, there exists a strong negative relationship 

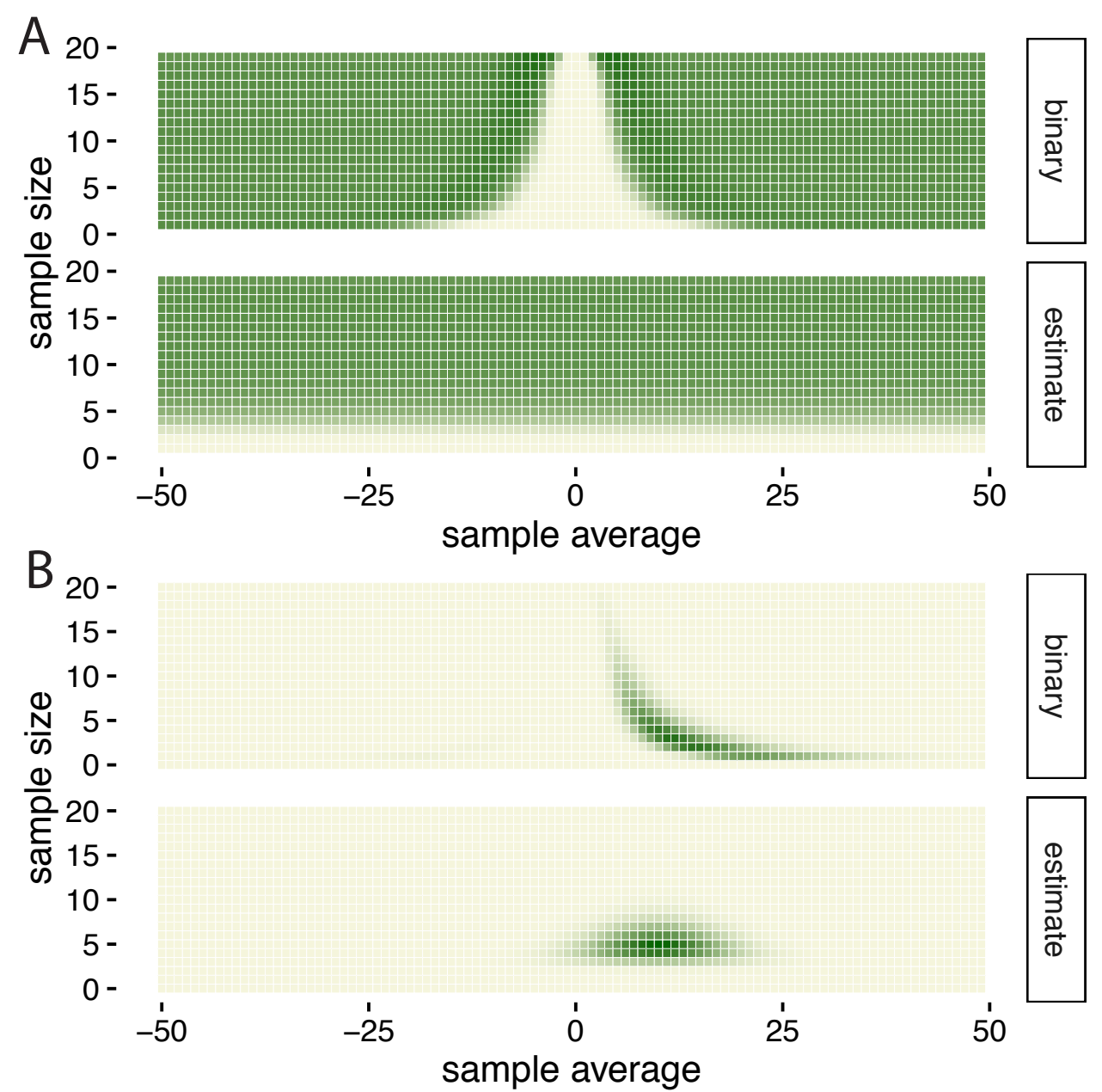

Figure 12. Predictions of the optimal stopping model with Gaussian outcomes. (A) Probability of continuing (green) or stopping (white) given observed data (current sample average and current sample size). (B) Probability of ending up with every possible final sample, assuming a true mean $\mu=10$.

under the binary goal, while they are independent under the estimation goal, for which the sample average is always the true underlying mean of the distribution (10).

\section{References}

Barr, D. J., Levy, R., Scheepers, C., \& Tily, H. J. (2013). Random effects structure for confirmatory hypothesis testing: Keep it maximal. Journal of memory and language, 68(3), 255-278.

Bogacz, R., Brown, E., Moehlis, J., Holmes, P., \& Cohen, J. D. (2006). The physics of optimal decision making: a formal analysis of models of performance in twoalternative forced-choice tasks. Psychological review, 113(4), 700.

Bogacz, R., Hu, P. T., Holmes, P. J., \& Cohen, J. D. (2010). Do humans produce the speed-accuracy trade-off that maximizes reward rate? The Quarterly Journal of Experimental Psychology, 63(5), 863-891.

Busemeyer, J. R., \& Rapoport, A. (1988). Psychological models of deferred decision making. Journal of Mathematical Psychology, 32(2), 91-134.

Denrell, J. (2003). Vicarious learning, undersampling of failure, and the myths of management. Organization Science, 14(3), 227-243.

Denrell, J. (2005). Why most people disapprove of me: experience sampling in impression formation. Psychological review, 112(4), 951.

Edwards, W. (1965). Optimal strategies for seeking information: Models for statistics, choice reaction times, and human information processing. Journal of Mathematical Psychology, 2(2), 312-329.

Ferguson, T. S. (n.d.). Optimal stopping and applications.

Fiedler, K. (2000). Beware of samples! a cognitiveecological sampling approach to judgment biases. Psy- 


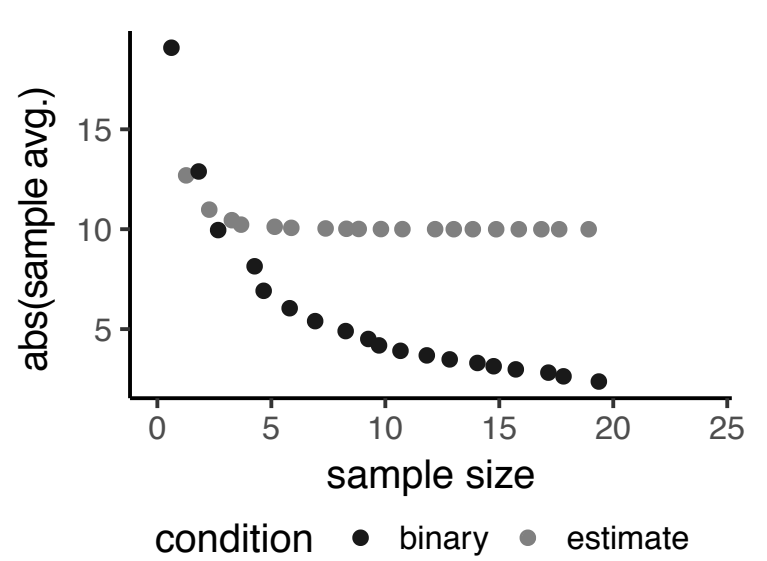

Figure 13. Relationship between sample size and absolute sample average (distance from the binary criterion, 0 ), based on the sampling goal of the optimal learner. Again, larger samples have less extreme averages under the binary sampling regime. Note that, the first two data points of the estimation condition lie slightly above the true sample mean (10), due to the fact that the model was run on discretized values.

chological review, 107(4), 659.

Fiedler, K. (2008). The ultimate sampling dilemma in experience-based decision making. Journal of experimental psychology. Learning, memory, and cognition, 34(1), 186-203. Retrieved from http: //www .ncbi.nlm.nih.gov/pubmed/18194062 doi: $10.1037 / 0278-7393.34 .1 .186$

Fiedler, K., \& Juslin, P. (Eds.). (2006). Information sampling and adaptive cognition. Cambridge, UK: Cambridge University Press.

Francis, G. (2012). Publication bias and the failure of replication in experimental psychology. Psychonomic bulletin $\mathcal{F}$ review, 19(6), 975-991. doi: 10.3758/s13423012-0322-y

Garthwaite, P. H., Kadane, J. B., \& O’Hagan, A. (2005). Statistical methods for eliciting probability distributions. Journal of the American Statistical Association, 100(470), 680-700.

Goldstein, D. G., \& Gigerenzer, G. (2002). Models of ecological rationality: The recognition heuristic. Psychological Review, 109(1), 75-90.

Griffin, D., \& Tversky, A. (1992). The weighing of evidence and the determinants of confidence. Cognitive psychology, 24(3), 411-435.

Hertwig, R., \& Pleskac, T. J. (2010). Decisions from experience: Why small samples? Cognition, 115(2), 225237.

Juni, M. Z., Gureckis, T. M., \& Maloney, L. T. (2016). Information sampling behavior with explicit sampling costs. Decision, 3(3), 147.
Kahneman, D., \& Tversky, A. (1972). Subjective probability: A judgment of representativeness. Cognitive psychology, 3(3), 430-454.

Kareev, Y., Arnon, S., \& Horwitz-Zeliger, R. (2002). On the misperception of variability. Journal of Experimental Psychology: General, 131(2), 287.

Kvam, P. D., \& Pleskac, T. J. (2016). Strength and weight: The determinants of choice and confidence. Cognition, 152, 170-180.

Murphy, A. H., \& Winkler, R. L. (1977). Reliability of subjective probability forecasts of precipitation and temperature. Applied Statistics, 41-47.

Nosofsky, R. M., \& Palmeri, T. J. (1997). An exemplarbased random walk model of speeded classification. Psychological review, 104(2), 266.

Pachur, T., Hertwig, R., \& Rieskamp, J. (2013). The mind as an intuitive pollster: Frugal search in social spaces. Simple heuristics in a social world, 261-91.

Peterson, C. R., Snapper, K. J., \& Murphy, A. H. (1972). Credible interval temperature forecasts. Bulletin of the American Meteorological Society, 53(10), 966-970.

Pleskac, T. J., Busemeyer, J. R., \& Others. (2010). Two-stage dynamic signal detection: a theory of choice, decision time, and confidence. Psychological review, 117(3), 864. doi: $10.1037 / \mathrm{a} 0019737$

Ratcliff, R. (1978). A theory of memory retrieval. Psychological review, 85(2), 59.

Ratcliff, R., \& Rouder, J. N. (1998). Modeling response times for two-choice decisions. Psychological Science, 9(5), 347-356.

Seale, D. A., \& Rapoport, A. (1997). Sequential decision making with relative ranks: An experimental investigation of the" secretary problem". Organizational behavior and human decision processes, 69(3), 221236.

Simmons, J. P., Nelson, L. D., \& Simonsohn, U. (2011). False-Positive Psychology: Undisclosed Flexibility in Data Collection and Analysis Allows Presenting Anything as Significant. Psychological Science, 22(11), 1359-1366. doi: 10.1177/0956797611417632

Tversky, A., \& Edwards, W. (1966). Information versus reward in binary choices. Journal of Experimental Psychology, 71(5), 680.

Tversky, A., \& Kahneman, D. (1971). Belief in the law of small numbers. Psychological Bulletin; Psychological Bulletin, 76(2), 105.

Unkelbach, C., Fiedler, K., \& Freytag, P. (2007). Information repetition in evaluative judgments: Easy to monitor, hard to control. Organizational Behavior and Human Decision Processes, 103(1), 37-52.

Usher, M., \& McClelland, J. L. (2001). The time course of perceptual choice: the leaky, competing accumulator model. Psychological review, 108(3), 550. 
Winkler, R. (1967). The Quantification of Judgement: Some methodological Suggestions. Journal of the American
Statistical Association, 62(320), 1105-1120. 